\title{
In Silco Molecular Modeling of Chalcone Based Aryloxyethylamines as Antihyperglycemic Agents
}

Poonam Shukla $^{1 *}$, Ram Pratap ${ }^{2}$, Shalini Singh ${ }^{3}$, Neha Rahuja ${ }^{4}$, Ashok K Prasad ${ }^{1}$ and Arvind Kumar Shrivastava ${ }^{4}$

${ }^{1}$ Department of Chemistry, University of Delhi, Delhi, India

${ }^{2}$ Medicinal and Process Chemistry Division, Central Drug Research Institute, Lucknow, Uttar Pradesh, India

${ }^{3}$ QSAR and Cheminformatics Laboratory, Department of Chemistry, Bareilly College, Bareilly, Uttar Pradesh, India

${ }^{4}$ Department of Biochemistry, Central Drug Research Institute, Lucknow, Uttar Pradesh, India

\begin{abstract}
Despite enormous efforts have been made in the search for new drugs, diabetes mellitus (DM) still remains the cause of mortality worldwide. A series of chalcone based aryloxyethylamines were synthesized and evaluated for their anti-hyperglycemic activity in SLM and STZ rat models. The majority of the compounds exhibited temperate to good activity ranging from $18.0 \%$ to $39.8 \%$ in SLM and $14.6 \%$ to $31.5 \%$ in STZ models, respectively. The most potent compound 31 exhibited glucose lowering of $27.0 \%$ in SLM and $31.5 \%$ in STZ models. A definite structure-activity relationship was observed while varying the nature and position of the different amines in ring-A of chalcone.
\end{abstract}

Keywords: Chalcone; Antihyperglycemic; Aryloxyethylamine; Oxidative stress; Antioxidant

\section{Introduction}

Diabetes mellitus, an assembly of metabolic diseases in which the patient has elevated blood glucose, either because of insufficient insulin production or because the proper non-responsibility of body's cells to insulin, or both. NIDDM is a chaos characterized by insulin resistance, hyperglycemia and hyperinsulinaemia, frequently associated with obesity, dyslipidemia and hypertension foremost to cardiovascular risks [1]. In such cases, compensation of normal adipose tissue levels argues in mitigation of the insulin resistant state [2]. Diminution of body fat growth via diet and exercise is generally the first treatment for diabetes with the link between obesity and type-II diabetes. The antiobesity drugs based therapies for NIDDM, are targeted at a lessening of energy intake or absorption (anorectic drugs) but an increase in energy expenditure (thermogenic drugs) serve as an gorgeous substitute for the treatment of obesity and hence, diabetes. Induction of thermogenesis is either through stimulation of nuclear receptor of PPAR family [3] or adrenergic receptor (AR) in membrane of adipose tissue [4].

In 1980 s, $\beta 3$-adrenergic receptor was revealed on the cell surface of both white and brown adipose tissues. $\beta_{3}$-AR agonists were observed to concurrently augment lipolysis, fat oxidation, energy outlay [5] and insulin action foremost to the belief that, this receptor might afford as an attractive target for the treatment of diabetes and obesity [6]. Arylethanolamines and aryloxypropanolamines were first reported as $\beta_{3}$-AR agonists earlier [7]. CL-316,243 is the most selective $\beta_{3}$-AR agonist tested [8]

Troglitazone is also an antidiabetic drug of thiazolidinedione (I) class having unique structural feature [9], (Figure 1) is the presence of an antioxidant moiety of vitamin-E mutually with a PPAR- $\gamma$ nuclear receptor motivating pharmacophore in a single molecule. It is however failed with toxic metabolites but provided a source for further scheming molecules for multi-factorial diseases. Oxidative stress plays a vital role in diabetic patients by macrovascular complications through Maillard reaction [10]. The chalcones with antioxidant properties having plant origin have attracted our attention to explore hybrid structures with them for antidiabetic activity. Chalcones are natural products having various biological activities including antioxidant [11], antimalarial [12], antileishmanial [13], anti-inflammatory [14] and antitumor [15]. The antioxidative nature of chalcones might be one of the factors for different activities. In this ongoing programme, we have synthesized two series of chalcone based aryloxypropanolamines (II) [16] (Figure 1) have shown a potent anti-diabetic activity.

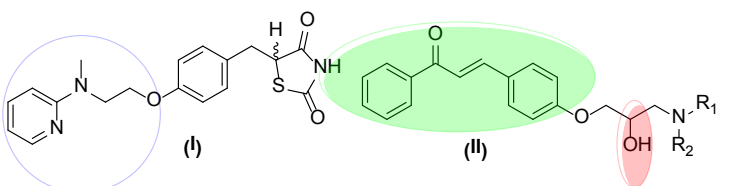

Figure 1: Proptotypes of thiazolidinones and chalcones.

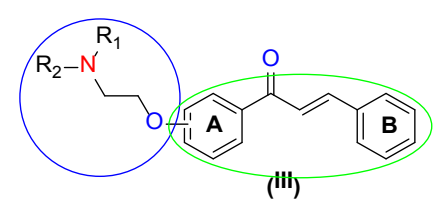

Figure 2: Prototype structure of synthesized molecules.

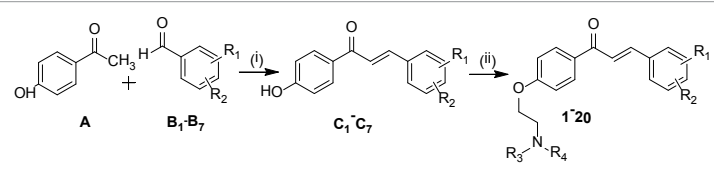

Scheme 1: Reagents: (i) Aq. $\mathrm{NaOH}$, methanol, r. t., 6-8 hr; (ii) $\mathrm{N}$, $\mathrm{N}$-dialkylethylaminochloride, dried $\mathrm{K}_{2} \mathrm{CO}_{3}$, dry acetone.

After biological evaluation of those molecules against diabetic rats and finding a potential activity, a different series of chalcone based molecules have been synthesized by replacing the $\mathrm{CHOH}$ group from aryloxypropanolamine forming an aryloxyethylamines on ring-A of the basic moiety. The synthesized new prototype-III (Figure 2) have chalcone with pharmacophore which is used in different types of drugs as a side chain having property to increase lipophilicity. Most of the

*Corresponding author: Poonam Shukla, Department of Chemistry University of Delhi, Delhi, India, Tel: 7827963916; Fax: 7827963916; E-mail: poonamshukla.du@gmail.com

Received July 22, 2017; Accepted July 28, 2017; Published July 31, 2017

Citation: Shukla P, Pratap R, Singh S, Rahuja N, Prasad AK, et al. (2017) In Silco Molecular Modeling of Chalcone Based Aryloxyethylamines as Antihyperglycemic Agents. Med Chem (Los Angeles) 7: 226-234. doi: 10.4172/2161-0444.1000461

Copyright: $\odot 2017$ Shukla $P$, et al. This is an open-access article distributed under the terms of the Creative Commons Attribution License, which permits unrestricted use, distribution, and reproduction in any medium, provided the original author and source are credited. 
Citation: Shukla P, Pratap R, Singh S, Rahuja N, Prasad AK, et al. (2017) In Silco Molecular Modeling of Chalcone Based Aryloxyethylamines as Antihyperglycemic Agents. Med Chem (Los Angeles) 7: 226-234. doi: 10.4172/2161-0444.1000461

\begin{tabular}{|c|c|c|c|c|}
\hline \multirow{2}{*}{ Compounds } & \multirow{2}{*}{ Chemical Structure } & \multirow{2}{*}{$\%$ Improvement SLM } & \multicolumn{2}{|c|}{ \% Lowering (STZ) } \\
\hline & & & $5 \mathrm{hr}$ & $24 \mathrm{hr}$ \\
\hline 1 & & +17.5 & ND & ND \\
\hline 2 & & 2.65 & ND & ND \\
\hline 3 & & $23.8^{*}$ & 5.63 & $17.6^{*}$ \\
\hline 4 & & 17.2 & ND & ND \\
\hline 5 & & $25.2^{*}$ & 11.7 & 14.0 \\
\hline 6 & & 11.3 & ND & ND \\
\hline 7 & & $21.3^{*}$ & $20.8^{* *}$ & $18.0^{* *}$ \\
\hline 8 & & $18.8^{*}$ & 13.4 & 6.55 \\
\hline 9 & & $20.5^{*}$ & $14.8^{*}$ & 9.71 \\
\hline 10 & & $19.1^{*}$ & 11.0 & 12.2 \\
\hline 11 & & $18.0^{*}$ & $14.8^{*}$ & $20.4^{*}$ \\
\hline 12 & & $20.7^{*}$ & $21.9^{*}$ & $23.2^{*}$ \\
\hline 13 & & $25.8^{\star *}$ & $17.9^{*}$ & $16.0^{*}$ \\
\hline 14 & & $24.9^{* *}$ & $27.6^{* *}$ & $23.1^{* *}$ \\
\hline 15 & & $18.5^{*}$ & $15.0^{*}$ & $22.6^{*}$ \\
\hline 16 & & $22.8^{* *}$ & $14.6^{*}$ & $14.3^{*}$ \\
\hline 17 & & $19.6^{*}$ & $29.4^{* *}$ & $25.0^{\star \star}$ \\
\hline
\end{tabular}




\begin{tabular}{|c|c|c|c|}
\hline 18 & $19.0^{*}$ & 12.1 \\
\hline 20 & $27.0^{* *}$ & 13.2 \\
\hline SD & $24.6^{* *}$ & $25.0^{* *}$ \\
\hline
\end{tabular}

Table 1: Effect of synthetic Compounds and standard drug metformin on glucose tolerance post sucrose load in normal rats and decline in blood glucose level in streptozotocin induced diabetic rats.

compounds of this new series have shown better anti-hyperglycemic activity than earlier series.

\section{Chemistry}

Synthesis of chalcone derived, ring-A substituted aryloxyethylamines (1-20)

The chalcones were synthesized with the help of Claisen-Schmidt condensation, which has been reported above. 4-hydroxy-acetophenone (A) and appropriate amount of substituted benzaldehydes $\left(\mathbf{B}_{1}-\mathbf{B}_{7}\right)$ were reacted using aqueous sodium hydroxide in methanol at room temperature to provide corresponding hydroxy-chalcones $\left(\mathbf{C}_{1}-\mathbf{C}_{7}\right)$. These chalcones were reacted with $\mathrm{N}, \mathrm{N}$-dialkylaminoethylchloride in presence of dried $\mathrm{K}_{2} \mathrm{CO}_{3}$ (4-5eq.) in acetone to get products 1-20 (Scheme 1).

\section{Results and Discussion}

Anti-hyperglycemic activity of chalcone derived aryloxyethylamines

These molecules were evaluated for anti-hyperglycemic activity on both SLM and STZ models which have shown moderate to good potential as anti-hyperglycemic agents presented below (Table 1).

Effect of compounds and standard drug metformin on improvement of glucose tolerance on normal rats (SLM)

A set of 20 substituted chalcones with different functionalities at $\mathrm{R}_{1}$ and $\mathrm{R}_{2}$ were synthesized in the Medicinal and Process Development Chemistry of the Institute. These were first evaluated for improvement in glucose tolerance in normal rats. The results obtained from the variance analysis showed in Table 1, depict the percentage improvement profile of 20 test compounds (1-20) and standard drug Metformin. Among these 20 compounds, most of the compounds i.e., $3,5,7,8,9,10,11,12,13,14,15,16,17,18,19$ and 20 showed significant effect were calculated to be around 23.8, 25.2, 21.3, 18.8, 20.5, 19.1, 18.0, 20.7, 25.8, 24.9, 18.5, 22.8, 19.6, 19.0, 27.0 and $24.6 \%$ improvement in glucose tolerance respectively on blood glucose level of normoglycemic rats while the standard drug metformin treated group showed $\mathbf{3 5 . 6 \%}$ improvement in SLM. The rest of the compounds did not cause any detectable improvement on the blood glucose level of normo-glycaemic rats.

\section{Effect of compounds and standard drug metformin on decline in blood glucose level of STZ treated diabetic rats}

Those compounds which showed significant improvement in glucose tolerance in normal rats were further tested on low dosed Streptozotocin induced diabetic rats for their anti-hyperglycemic activity evaluation. Among the tested compounds i.e., 3, 5, 7, 8, 9, 10, $11,12,13,14,15,16,17,18,19$ and 20 , compound code $7,9,11,12,13$, $14,15,16,17,19$ and 20 caused significant decline of 20.8, 14.8, 14.8, 21.9, 17.9, 27.6, 15.0, 14.6, 29.4, 31.5, 17.1\% during $5 \mathrm{hr}$ and 18.0, 9.71, 20.4, 23.2, 16.0, 23.1, 22.6, 14.3, 25.0 25.0 and 9.74\% during 24 hr respectively whereas Compound code $3,5,6,8,10$ and 18 showed very mild effect on STZ treated rats. The standard drug Metformin at the dose level of $100 \mathrm{mg} / \mathrm{kg}$ showed a blood glucose lowering effect of $\mathbf{2 8 . 9 \%}$ and $\mathbf{2 5 . 7 \%}$ after $5 \mathrm{hr}$ and $24 \mathrm{hr}$ intervals respectively.

\section{Experimrental Data}

\section{Chemistry}

4'-(2-Dimethylamino-ethoxy)-3, 4-methelinedioxy-chalcone (1): To a solution of 4-hydroxy-chalcone $\mathrm{E}_{1}(0.5 \mathrm{~g}, 1.86 \mathrm{mmol})$ in dry acetone was added dried $\mathrm{K}_{2} \mathrm{CO}_{3}$ (3eq.) of and 2-chloroethyl-dimethylamine $(0.22 \mathrm{~g}, 2.14 \mathrm{mmol})$. This reaction mixture was refluxed for $7-8 \mathrm{~h}$ rs at $70^{\circ} \mathrm{C}$ to get 14 . Solid, Yield: $27 \%$, m. p.: $103^{\circ} \mathrm{C}$, MS: $340(\mathrm{M}+1)$, IR(KBr): 3415, 1600, 449, ${ }^{1} \mathrm{HNMR}\left(300 \mathrm{MHz}, \mathrm{CDCl}_{3}\right), \delta 7.15(\mathrm{~d}, \mathrm{~J}=$ $11.25 \mathrm{~Hz}, 2 \mathrm{H}$ 2', 6'-H), $7.73(\mathrm{~d}, \mathrm{~J}=15.51 \mathrm{~Hz}, 1 \mathrm{H}, \beta-\mathrm{H}), 7.39(\mathrm{~d}, \mathrm{~J}=$ $15.48 \mathrm{~Hz}, 1 \mathrm{H}, \alpha-\mathrm{H}), 7.17(\mathrm{~s}, 1 \mathrm{H}, 2-\mathrm{H}), 7.13(\mathrm{~d}, \mathrm{~J}=8.01 \mathrm{~Hz}, 1 \mathrm{H}, 6-\mathrm{H})$, $7.00(\mathrm{~d}, \mathrm{~J}=8.76 \mathrm{~Hz}, 2 \mathrm{H}, 3$ ', 5'-H), $6.85(\mathrm{~d}, \mathrm{~J}=8.01 \mathrm{~Hz}, 1 \mathrm{H}, 5-\mathrm{H}), 6.03(\mathrm{~s}$, $\left.2 \mathrm{H}, \mathrm{OCH}_{2} \mathrm{O}\right), 4.15\left(\mathrm{t}, 2 \mathrm{H}, \mathrm{OCH}_{2}\right), 2.769\left(\mathrm{t}, 2 \mathrm{H},-\mathrm{NCH}_{2}\right), 1.62,(\mathrm{~s}, 6 \mathrm{H}$, $\left.-\mathrm{NCH}_{3}\right)$.

4'-(2-Diethylamino-ethoxy)-3, 4-methelinedioxy-chalcone (2): To a solution of 4-hydroxy-chalcone $\mathrm{E}_{1}(0.5 \mathrm{~g}, 1.86 \mathrm{mmol})$ in dry acetone was added dried $\mathrm{K}_{2} \mathrm{CO}_{3}$ (5eq.) and 2-chloroethyl-diethylamine $(0.25 \mathrm{~g}, 2.18 \mathrm{mmol})$. This reaction mixture was refluxed for 8-9 hrs at $70^{\circ} \mathrm{C}$ to get 15 . Solid, Yield: $5 \%$, m. p.: $105^{\circ} \mathrm{C}$, MS: $368(\mathrm{M}+1)$, IR $(\mathrm{KBr})$ : 3020, 1516, 416, 767, ${ }^{1} \mathrm{HNMR}\left(200 \mathrm{MHz}, \mathrm{CDCl}_{3}\right), \delta 8.02(\mathrm{~d}, \mathrm{~J}=8.82 \mathrm{~Hz}$, $2 \mathrm{H}$ 2', 6'-H), $7.73(\mathrm{~d}, \mathrm{~J}=15.52 \mathrm{~Hz}, 1 \mathrm{H}, \beta-\mathrm{H}), 7.38(\mathrm{~d}, \mathrm{~J}=15.50 \mathrm{~Hz}, 1 \mathrm{H}$, $\alpha-\mathrm{H}), 7.17(\mathrm{~s}, 1 \mathrm{H}, 2-\mathrm{H}), 7.13(\mathrm{~d}, \mathrm{~J}=8.08 \mathrm{~Hz}, 1 \mathrm{H}, 6-\mathrm{H}), 6.15(\mathrm{~d}, \mathrm{~J}=8.82$ $\mathrm{Hz}, 2 \mathrm{H}, 3$ ', 5'-H), 6.84 (d, J = 7.15 Hz, 1H, 5-H), $6.03\left(\mathrm{~s}, 2 \mathrm{H}, \mathrm{OCH}_{2} \mathrm{O}\right)$, $4.13\left(\mathrm{t}, 2 \mathrm{H}, \mathrm{OCH}_{2}\right), 2.7\left(\mathrm{t}, 2 \mathrm{H},-\mathrm{NCH}_{2}\right), 2.27\left(\mathrm{q}, 4 \mathrm{H}, \mathrm{NCH}_{2}\right), 1.08(\mathrm{t}, 6 \mathrm{H}$, $\left.\mathrm{CH}_{3}\right)$.

4'-(2-Pyrrolidin-1-yl-ethoxy)-3, 4-methelinedioxy-chalcone (3): To a solution of 4-hydroxy-chalcone $\mathrm{E}_{1}(0.3 \mathrm{~g}, 1.12 \mathrm{mmol})$ in dry acetone was added dried $\mathrm{K}_{2} \mathrm{CO}_{3}$ (4eq.) and 2-chloroethyl-pyrrolidine $(0.15$ g, $1.25 \mathrm{mmol})$. This reaction mixture was refluxed for $9-10 \mathrm{hrs}$ at $70^{\circ} \mathrm{C}$ to get 16 . Solid, Yield: $73.17 \%$, m. p.: $140^{\circ} \mathrm{C}$, MS (ESI): $366(\mathrm{M}+1)$, IR (KBr): 3380, 2361, 1515, 416, 670, ${ }^{1} \mathrm{HNMR}\left(300 \mathrm{MHz}, \mathrm{CDCl}_{3}\right), \delta 8.03$ $(\mathrm{d}, \mathrm{J}=8.79 \mathrm{~Hz}, 2 \mathrm{H}$ 2', 6'-H), $7.74(\mathrm{~d}, \mathrm{~J}=15.54 \mathrm{~Hz}, 1 \mathrm{H}, \beta-\mathrm{H}), 7.40$ (d, J $=15.54 \mathrm{~Hz}, 1 \mathrm{H}, \alpha-\mathrm{H}), 7.19(\mathrm{~s}, 1 \mathrm{H}, 2-\mathrm{H}), 7.14(\mathrm{~d}, \mathrm{~J}=8.07 \mathrm{~Hz}, 1 \mathrm{H}, 6-\mathrm{H})$, $7.01(\mathrm{~d}, \mathrm{~J}=8.85 \mathrm{~Hz}, 2 \mathrm{H}, 3$ ', 5'-H), $6.84(\mathrm{~d}, \mathrm{~J}=8.61 \mathrm{~Hz}, 1 \mathrm{H}, 5-\mathrm{H}), 6.05$ $\left(\mathrm{s}, 2 \mathrm{H}, \mathrm{OCH}_{2} \mathrm{O}\right), 4.19\left(\mathrm{t}, 2 \mathrm{H}, \mathrm{OCH}_{2}\right), 2.11\left(\mathrm{t}, 2 \mathrm{H},-\mathrm{NCH}_{2}\right), 2.67-2.27(\mathrm{~m}$, $\left.4 \mathrm{H},-\mathrm{NCH}_{2}\right), 1.87-1.82\left(\mathrm{~m}, 4 \mathrm{H}, \mathrm{CH}_{2}\right.$ - 
Citation: Shukla P, Pratap R, Singh S, Rahuja N, Prasad AK, et al. (2017) In Silco Molecular Modeling of Chalcone Based Aryloxyethylamines as Antihyperglycemic Agents. Med Chem (Los Angeles) 7: 226-234. doi: 10.4172/2161-0444.1000461

4'-(2-Piperidin-1-yl-ethoxy)-3,4 methelinedioxy-chalcone (4): To a solution of 4-hydroxy-chalcone $\mathrm{E}_{1}(0.3 \mathrm{~g}, 1.12 \mathrm{mmol})$ in dry acetone was added dried $\mathrm{K}_{2} \mathrm{CO}_{3}$ (4eq.) of and 2-chloroethyl-piperidine $(0.47 \mathrm{~g}, 1.35 \mathrm{mmol})$. This reaction mixture was refluxed for $8-9 \mathrm{hrs}$ at $70^{\circ} \mathrm{C}$ to get 17. Solid, Yield: $76.19 \%$, m. p.: $42^{\circ} \mathrm{C}$, MS (ESI): $380(\mathrm{M}+1)$, IR (KBr): 3380, 1516, 448, 668, ${ }^{1} \mathrm{HNMR}\left(300 \mathrm{MHz}, \mathrm{CDCl}_{3}\right), \delta 8.03(\mathrm{~d}$, $\left.\mathrm{J}=8.82 \mathrm{~Hz}, 2 \mathrm{H} 2^{\prime}, 6^{\prime}-\mathrm{H}\right), 7.74(\mathrm{~d}, \mathrm{~J}=15.51 \mathrm{~Hz}, 1 \mathrm{H}, \beta-\mathrm{H}), 7.40(\mathrm{~d}, \mathrm{~J}=$ $15.54 \mathrm{~Hz}, 1 \mathrm{H}, \alpha-\mathrm{H}), 7.19$ (s, 1H, 2-H), $7.17(\mathrm{~d}, \mathrm{~J}=8.58 \mathrm{~Hz}, 1 \mathrm{H}, 6-\mathrm{H})$, $7.00(\mathrm{~d}, \mathrm{~J}=8.85 \mathrm{~Hz}, 2 \mathrm{H}, 3$ ', 5'-H), $6.86(\mathrm{~d}, \mathrm{~J}=7.15 \mathrm{~Hz}, 1 \mathrm{H}, 5-\mathrm{H}), 6.04$ $\left(\mathrm{s}, 2 \mathrm{H}, \mathrm{OCH}_{2} \mathrm{O}\right), 4.19\left(\mathrm{t}, 2 \mathrm{H}, \mathrm{OCH}_{2}\right), 2.82\left(\mathrm{t}, 2 \mathrm{H},-\mathrm{NCH}_{2}\right), 2.56-2.52(\mathrm{~m}$, $\left.4 \mathrm{H},-\mathrm{NCH}_{2}\right), 1.66-1.60\left(\mathrm{~m}, 6 \mathrm{H}, \mathrm{CH}_{2}\right)$.

4'-(2-Azepan-1-yl-ethoxy)-3, 4-methelinedioxy-chalcone (5): To a solution of 4-hydroxy-chalcone $\mathrm{E}_{1}(0.3 \mathrm{~g}, 1.12 \mathrm{mmol})$ in dry acetone was added dried $\mathrm{K}_{2} \mathrm{CO}_{3}$ (3eq.) and 2-chloroethyl-azepane (0.18 g, 1.25 $\mathrm{mmol})$. This reaction mixture was refluxed for $7-8 \mathrm{hrs}$ at $70^{\circ} \mathrm{C}$ to get 18 . Solid, Yield: $7.8 \%$, m. p.: $45^{\circ} \mathrm{C}$, MS (ESI): $311(\mathrm{M}+1)$, IR (KBr): 3415 , 290, 1515, 448, ${ }^{1} \mathrm{HNMR}\left(300 \mathrm{MHz}, \mathrm{CDCl}_{3}\right), \delta 8.02(\mathrm{~d}, \mathrm{~J}=8.84 \mathrm{~Hz}, 2 \mathrm{H}$, 2', 6'-H), $7.73(\mathrm{~d}, \mathrm{~J}=15.50 \mathrm{~Hz}, 1 \mathrm{H}, \beta-\mathrm{H}), 7.39(\mathrm{~d}, \mathrm{~J}=15.52 \mathrm{~Hz}, 1 \mathrm{H}$, $\alpha-\mathrm{H}), 7.17(\mathrm{~s}, 1 \mathrm{H}, 2-\mathrm{H}), 7.13(\mathrm{~d}, \mathrm{~J}=8.16 \mathrm{~Hz}, 1 \mathrm{H}, 6-\mathrm{H}), 6.15(\mathrm{~d}, \mathrm{~J}=8.88$ $\mathrm{Hz}, 2 \mathrm{H}, 3$ ', 5'-H), 6.85 (d, J = 7.13 Hz, 1H, 5-H), 6.03 (s, 2H, $\mathrm{OCH}_{2} \mathrm{O}$ ), $4.15\left(\mathrm{t}, 2 \mathrm{H}, \mathrm{OCH}_{2}\right), 2.15\left(\mathrm{t}, 2 \mathrm{H},-\mathrm{NCH}_{2}\right), 2.79\left(\mathrm{~m}, 4 \mathrm{H},-\mathrm{NCH}_{2}\right), 1.27(\mathrm{~m}$, $\left.8 \mathrm{H}, \mathrm{CH}_{2}\right)$.

4'-(2-Morpholin-4-yl-ethoxy)-3, 4-methelinedioxy-chalcone 6): To a solution of 4-hydroxy-chalcone $\mathrm{E}_{1}(0.3 \mathrm{~g}, 1.12 \mathrm{mmol})$ in dry acetone was added dried $\mathrm{K}_{2} \mathrm{CO}_{3}$ (5eq.) of and 2-chloroethyl-morpholine $(0.168$ g, $1.30 \mathrm{mmol})$. This reaction mixture was refluxed for $9-10 \mathrm{hrs}$ at $70^{\circ} \mathrm{C}$ to get 19 . Solid, Yield: $62.79 \%$, m. p.: $135^{\circ} \mathrm{C}$, MS (ESI): $382(\mathrm{M}+1)$, IR (KBr): 3378, 1515, 416, 670, ${ }^{1} \mathrm{HNMR}\left(300 \mathrm{M} \mathrm{Hz}, \mathrm{CDCl}_{3}\right), \delta 8.04$ (d, J = $\left.8.85 \mathrm{~Hz}, 2 \mathrm{H} \mathrm{2}, 6^{\prime}-\mathrm{H}\right), 7.74(\mathrm{~d}, \mathrm{~J}=15.51 \mathrm{~Hz}, 1 \mathrm{H}, \beta-\mathrm{H}), 7.40(\mathrm{~d}, \mathrm{~J}=15.48$ $\mathrm{Hz}, 1 \mathrm{H}, \alpha-\mathrm{H}), 7.19$ (s, 1H, 2-H), $7.17(\mathrm{~d}, \mathrm{~J}=8.43 \mathrm{~Hz}, 1 \mathrm{H}, 6-\mathrm{H}), 7.00$ $\left(\mathrm{d}, \mathrm{J}=8.79 \mathrm{~Hz}, 2 \mathrm{H}, 3^{\prime}, 5^{\prime}-\mathrm{H}\right), 6.9(\mathrm{~d}, \mathrm{~J}=8.82 \mathrm{~Hz}, 1 \mathrm{H}, 5-\mathrm{H}), 6.05(\mathrm{~s}, 2 \mathrm{H}$, $\left.\mathrm{OCH}_{2} \mathrm{O}\right), 4.20\left(\mathrm{t}, 2 \mathrm{H}, \mathrm{OCH}_{2}\right), 3.78-3.73\left(\mathrm{~m}, 4 \mathrm{H},-\mathrm{C}_{2} \mathrm{OCH}_{2}\right), 2.85(\mathrm{t}$, $\left.2 \mathrm{H},-\mathrm{NCH}_{2}\right), 2.63-2.58\left(\mathrm{~m}, 4 \mathrm{H}, \underline{\mathrm{C}}_{2} \mathrm{NC}_{2}\right)$.

4'-(2-Diethylamino-ethoxy)-3, 5-dichloro-chalcone (7): To a solution of 4-hydroxy-chalcone $\mathrm{E}_{2}(0.5 \mathrm{~g}, 1.70 \mathrm{mmol})$ in dry acetone was added dried $\mathrm{K}_{2} \mathrm{CO}_{3}$ (3eq.) of and 2-chloroethyl-diethylamine $(0.24$ g, $2.09 \mathrm{mmol})$. This reaction mixture was refluxed for $8-10 \mathrm{hrs}$ at $70^{\circ} \mathrm{C}$ to get 40 . Solid, Yield: $6.86 \%$, m. p.: $105-106^{\circ} \mathrm{C}$, MS (ESI): $39,311\left(\mathrm{M}^{+}\right.$, $\mathrm{M}^{+2}$ ), IR (KBr): 3021, 1603, 416, 763, ${ }^{1} \mathrm{HNMR}\left(300 \mathrm{MHz}, \mathrm{CDCl}_{3}\right), \delta 8.02$ $\left(\mathrm{d}, \mathrm{J}=8.82 \mathrm{~Hz}, 2 \mathrm{H}, 2^{\prime}, 6^{\prime}-\mathrm{H}\right), 6.16(\mathrm{~d}, \mathrm{~J}=8.82 \mathrm{~Hz}, 2 \mathrm{H}, 3$ ', 5'-H), 7.5 (d, J $=1.53 \mathrm{~Hz}, 1 \mathrm{H}, 4-\mathrm{H}), 7.68(\mathrm{~d}, \mathrm{~J}=15.69 \mathrm{~Hz}, 1 \mathrm{H}, \beta-\mathrm{H}), 7.52(\mathrm{~d}, \mathrm{~J}=15.63$ $\mathrm{Hz}, 1 \mathrm{H}, \alpha-\mathrm{H}), 7.50-7.43(\mathrm{~m}, 2 \mathrm{H}, 2,6-\mathrm{H}), 4.13\left(\mathrm{t}, 2 \mathrm{H}, \mathrm{OCH}_{2}\right), 2.8(\mathrm{t}, 2 \mathrm{H}$, $\left.\mathrm{NCH}_{2}\right), 2.27\left(\mathrm{q}, 4 \mathrm{H}, \mathrm{CH}_{2}\right), 1.08\left(\mathrm{t}, 6 \mathrm{H}, \mathrm{CH}_{3}\right)$.

4'-(2-Piperidin-1-yl-ethoxy)-3, 5-dichloro-chalcone (8): To a solution of 4-hydroxy-chalcone $\mathrm{E}_{2}(0.5 \mathrm{~g}, 1.70 \mathrm{mmol})$ in dry acetone was added dried $\mathrm{K}_{2} \mathrm{CO}_{3}$ (3eq.) and 2-chloroethyl-piperidine $(0.260 \mathrm{~g}$, $2.02 \mathrm{mmol}$ ). This reaction mixture was refluxed for $8-9 \mathrm{hrs}$ at $70^{\circ} \mathrm{C}$ to get 21. Solid, Yield: 74.63\%, m. p.: 107-27C, MS (ESI): 404, $406\left(\mathrm{M}^{+}\right.$, $\mathrm{M}^{+2}$ ), IR (KBr): 3021, 1605, 416, 64, ${ }^{1} \mathrm{HNMR}\left(300 \mathrm{MHz}, \mathrm{CDCl}_{3}\right), \delta 8.02$ $\left(\mathrm{d}, \mathrm{J}=8.88 \mathrm{~Hz}, 2 \mathrm{H}, 2^{\prime}, 6^{\prime}-\mathrm{H}\right), 6.16$ (d, J = $\left.8.85 \mathrm{~Hz}, 2 \mathrm{H}, 3^{\prime}, 5^{\prime}-\mathrm{H}\right), 7.5$ (d, J = $1.68 \mathrm{~Hz}, 1 \mathrm{H}, 4-\mathrm{H}), 7.68(\mathrm{~d}, \mathrm{~J}=15.69 \mathrm{~Hz}, 1 \mathrm{H}, \beta-\mathrm{H}), 7.51(\mathrm{~d}, \mathrm{~J}=15.66 \mathrm{~Hz}$, $1 \mathrm{H}, \alpha-\mathrm{H}), 7.47(\mathrm{~m}, 2 \mathrm{H}, 2,6-\mathrm{H}), 4.19\left(\mathrm{t}, 2 \mathrm{H}, \mathrm{OCH}_{2}\right), 2.81\left(\mathrm{t}, 2 \mathrm{H}, \mathrm{NCH}_{2}\right.$ ), $2.52\left(\mathrm{~m}, 4 \mathrm{H}, \mathrm{NCH}_{2}\right), 1.62\left(\mathrm{~m}, 6 \mathrm{H}, \mathrm{CH}_{2}\right)$.

4'-(2-Azepan-1-yl-ethoxy)-3, 5-dichloro-chalcone (9): To a solution of 4-hydroxy-chalcone $\mathrm{E}_{2}(0.5 \mathrm{~g}, 1.70 \mathrm{mmol})$ in dry acetone was added dried $\mathrm{K}_{2} \mathrm{CO}_{3}$ (4eq.) and 2-chloroethyl-azepane (0.28 g, 1.85 $\mathrm{mmol})$. This reaction mixture was refluxed for $8-9 \mathrm{hrs}$ at $70^{\circ} \mathrm{C}$ to get 22 . Solid, Yield: $6.86 \%$, m. p.: $85-87^{\circ} \mathrm{C}$, MS (ESI): $418,420\left(\mathrm{M}^{+}, \mathrm{M}^{+2}\right)$, IR (KBr): 3021, 2359, 45, 64, ${ }^{1} \mathrm{HNMR}\left(200 \mathrm{MHz}, \mathrm{CDCl}_{3}\right), \delta 8.02$ (d, J = 8.88
$\left.\mathrm{Hz}, 2 \mathrm{H}, 2^{\prime}, 6^{\prime}-\mathrm{H}\right), 6.16\left(\mathrm{~d}, \mathrm{~J}=8.36 \mathrm{~Hz}, 2 \mathrm{H}, 3^{\prime}, 5^{\prime}-\mathrm{H}\right), 7.73(\mathrm{~s}, 1 \mathrm{H}, 4-\mathrm{H})$, $7.69(\mathrm{~d}, \mathrm{~J}=15.82 \mathrm{~Hz}, 1 \mathrm{H}, \beta-\mathrm{H}), 7.51(\mathrm{~d}, \mathrm{~J}=15.2 \mathrm{~Hz}, 1 \mathrm{H}, \mathrm{a}-\mathrm{H}), 7.52-7.46$ $(\mathrm{m}, 2 \mathrm{H}, 2,6-\mathrm{H}), 4.15\left(\mathrm{t}, 2 \mathrm{H}, \mathrm{OCH}_{2}\right), 2.16\left(\mathrm{t}, 2 \mathrm{H}, \mathrm{NCH}_{2}\right), 2.79-2.76(\mathrm{~m}$, $\left.4 \mathrm{H}, \mathrm{NCH}_{2}\right), 1.63\left(\mathrm{~s}, 8 \mathrm{H}, \mathrm{CH}_{2}\right)$.

4'-(2-Diethylamino-ethoxy)-4-chloro-chalcone (10): To a solution of 4-hydroxy-chalcone $\mathrm{E}_{3}(0.5 \mathrm{~g}, 1.10 \mathrm{mmol})$ in dry acetone was added of dried $\mathrm{K}_{2} \mathrm{CO}_{3}$ (3eq.) and 2-chloroethyl-diethylamine $(0.27$ g, $2.19 \mathrm{mmol})$. This reaction mixture was refluxed for $8-9 \mathrm{hrs}$ at $70^{\circ} \mathrm{C}$ to get 23 . Solid, Yield: $78.57 \%$, m. p.: $30-32^{\circ} \mathrm{C}$, MS (ESI): $358(\mathrm{M}+1)$, IR (KBr): 3020, 1600, 417, 64, ${ }^{1} \mathrm{HNMR}\left(300 \mathrm{MHz}, \mathrm{CDCl}_{3}\right), \delta 8.02(\mathrm{~d}, \mathrm{~J}=$ $\left.8.82 \mathrm{~Hz}, 2 \mathrm{H} 2,6^{\prime}-\mathrm{H}\right), 7.74(\mathrm{~d}, \mathrm{~J}=15.66 \mathrm{~Hz}, 1 \mathrm{H}, \beta-\mathrm{H}), 7.57(\mathrm{~d}, \mathrm{~J}=8.49$ $\mathrm{Hz}, 2 \mathrm{H}, 2,6-\mathrm{H}), 7.51(\mathrm{~d}, \mathrm{~J}=15.66 \mathrm{~Hz}, 1 \mathrm{H}, \alpha-\mathrm{H}), 7.39(\mathrm{~d}, \mathrm{~J}=8.46 \mathrm{~Hz}$, $2 \mathrm{H}, 3,5-\mathrm{H}), 6.16\left(\mathrm{~d}, \mathrm{~J}=8.82 \mathrm{~Hz}, 2 \mathrm{H}, 3,5^{\prime}-\mathrm{H}\right), 4.13\left(\mathrm{t}, 2 \mathrm{H}, \mathrm{OCH}_{2}\right), 2.7(\mathrm{t}$, $\left.2 \mathrm{H},-\mathrm{NCH}_{2}\right), 2.27\left(\mathrm{q}, 4 \mathrm{H},-\mathrm{NCH}_{2}\right), 1.08\left(\mathrm{t}, 6 \mathrm{H},-\mathrm{CH}_{3}\right)$.

4'-(2-Piperidin-1-yl-ethoxy)-4-chloro-chalcone (11): To a solution of 4-hydroxy-chalcone $\mathrm{E}_{3}(0.5 \mathrm{~g}, 1.10 \mathrm{mmol})$ in dry acetone was added dried $\mathrm{K}_{2} \mathrm{CO}_{3}$ (5eq.) and 2-chloroethyl-piperidine $(0.29 \mathrm{~g}$, $2.24 \mathrm{mmol}$ ). This reaction mixture was refluxed for $8-9 \mathrm{hrs}$ at $70^{\circ} \mathrm{C}$ to get 24. Solid, Yield: $88.23 \%$, m. p.: $48-50^{\circ} \mathrm{C}$, MS (ESI): $370(\mathrm{M}+1)$, IR (KBr): 3433, 1640, 64, ${ }^{1} \mathrm{HNMR}\left(300 \mathrm{M} \mathrm{Hz}, \mathrm{CDCl}_{3}\right), \delta 8.04(\mathrm{~d}, \mathrm{~J}=8.85$ $\left.\mathrm{Hz}, 2 \mathrm{H}, 2^{\prime}, 6^{\prime}-\mathrm{H}\right), 7.77(\mathrm{~d}, \mathrm{~J}=15.66 \mathrm{~Hz}, 1 \mathrm{H}, \beta-\mathrm{H}), 7.59(\mathrm{~d}, \mathrm{~J}=8.46 \mathrm{~Hz}$, $2 \mathrm{H}, 2,6-\mathrm{H}), 7.53(\mathrm{~d}, \mathrm{~J}=15.63 \mathrm{~Hz}, 1 \mathrm{H}, \alpha-\mathrm{H}), 7.41(\mathrm{~d}, \mathrm{~J}=8.49 \mathrm{~Hz}, 2 \mathrm{H}$, 3, 5-H), $7.01(\mathrm{~d}, \mathrm{~J}=8.88 \mathrm{~Hz}, 2 \mathrm{H}, 3,5$ '- $\mathrm{H}), 4.22\left(\mathrm{t}, 2 \mathrm{H}, \mathrm{OCH}_{2}\right), 2.84(\mathrm{t}$, $\left.2 \mathrm{H},-\mathrm{NCH}_{2}\right), 2.56-2.54\left(\mathrm{~m}, 4 \mathrm{H},-\mathrm{NCH}_{2}\right), 1.69-1.61\left(\mathrm{~m}, 6 \mathrm{H},-\mathrm{CH}_{2}\right)$.

4'-(2-Azepan-1-yl-ethoxy)-4-chloro-chalcone (12): To a solution of 4-hydroxy-chalcone $\mathrm{E}_{3}(0.5 \mathrm{~g}, 1.10 \mathrm{mmol})$ in dry acetone was added dried $\mathrm{K}_{2} \mathrm{CO}_{3}$ (4eq.) and 2-chloroethyl-azepane $(0.32 \mathrm{~g}, 2.05 \mathrm{mmol})$. This reaction mixture was refluxed for $8-9 \mathrm{hrs}$ at $70^{\circ} \mathrm{C}$ to get 25 . Solid, Yield: $80.73 \%$, m. p.: $28-30^{\circ} \mathrm{C}$, MS (ESI): $384(\mathrm{M}+1)$, IR (KBr): 3450 , $1608,670,{ }^{1} \mathrm{HNMR}\left(300 \mathrm{M} \mathrm{Hz}, \mathrm{CDCl}_{3}\right), \delta 8.03(\mathrm{~d}, \mathrm{~J}=8.85 \mathrm{~Hz}, 2 \mathrm{H} \mathrm{2}$, $\left.6^{\prime}-\mathrm{H}\right), 7.76(\mathrm{~d}, \mathrm{~J}=15.60 \mathrm{~Hz}, 1 \mathrm{H}, \beta-\mathrm{H}), 7.59(\mathrm{~d}, \mathrm{~J}=8.46 \mathrm{~Hz}, 2 \mathrm{H}, 2,6-\mathrm{H})$, $7.53(\mathrm{~d}, \mathrm{~J}=5.5 \mathrm{~Hz}, 1 \mathrm{H}, \mathrm{a}-\mathrm{H}), 7.40(\mathrm{~d}, \mathrm{~J}=8.46 \mathrm{~Hz}, 2 \mathrm{H}, 3,5-\mathrm{H}), 7.01(\mathrm{~d}, \mathrm{~J}=$ $\left.8.85 \mathrm{~Hz}, 2 \mathrm{H}, 3^{\prime}, 5^{\prime}-\mathrm{H}\right), 4.19\left(\mathrm{t}, 2 \mathrm{H}, \mathrm{OCH}_{2}\right), 3.02\left(\mathrm{t}, 2 \mathrm{H},-\mathrm{NCH}_{2}\right), 2.84-2.81$ $\left(\mathrm{m}, 4 \mathrm{H},-\mathrm{NCH}_{2}\right), 1.70-1.27\left(\mathrm{~m}, 8 \mathrm{H},-\mathrm{CH}_{2}\right)$.

4'-(2-Diethylamino-ethoxy)-4-methyl-chalcone (13): To a solution of 4-hydroxy-chalcone $\mathrm{E}_{4}(0.5 \mathrm{~g}, 2.11 \mathrm{mmol})$ in dry acetone was added dried $\mathrm{K}_{2} \mathrm{CO}_{3}$ (3eq.) and 2-chloroethyl-diethylamine $(0.29 \mathrm{~g}$, $2.25 \mathrm{mmol})$. This reaction mixture was refluxed for $8-9 \mathrm{hrs}$ at $70^{\circ} \mathrm{C}$ to get 26. Liquid, Yield: 77.43\%, MS (ESI): $338(\mathrm{M}+1)$, IR (Neat): 3414, $1605,64,{ }^{1} \mathrm{HNMR}\left(300 \mathrm{MHz}, \mathrm{CDCl}_{3}\right), \delta 8.04(\mathrm{~d}, \mathrm{~J}=8.79 \mathrm{~Hz}, 2 \mathrm{H}, 2$ ', 6'$\mathrm{H}), 7.81(\mathrm{~d}, \mathrm{~J}=15.63 \mathrm{~Hz}, 1 \mathrm{H}, \beta-\mathrm{H}), 7.56(\mathrm{~d}, \mathrm{~J}=6.39 \mathrm{~Hz}, 2 \mathrm{H}, 2,6-\mathrm{H})$, $7.52(\mathrm{~d}, \mathrm{~J}=13.15 \mathrm{~Hz}, 1 \mathrm{H}, \alpha-\mathrm{H}), 7.23(\mathrm{~d}, \mathrm{~J}=7.50 \mathrm{~Hz}, 2 \mathrm{H}, 3,5-\mathrm{H}), 6.16(\mathrm{~d}$, $\mathrm{J}=8.79 \mathrm{~Hz}, 2 \mathrm{H}, 3,5-\mathrm{H}), 4.16\left(\mathrm{t}, 2 \mathrm{H}, \mathrm{OCH}_{2}\right), 2.10\left(\mathrm{t}, 2 \mathrm{H}, \mathrm{CH}_{2} \mathrm{~N}\right), 2.70$ $\left(\mathrm{m}, 4 \mathrm{H}, \mathrm{NCH}_{2}\right), 2.41\left(\mathrm{~s}, 3 \mathrm{H}, \mathrm{CH}_{3}\right), 1.11\left(\mathrm{t}, 6 \mathrm{H}, \mathrm{CH}_{3}\right)$.

4'-(2-Piperidin-1-yl-ethoxy)-4-methyl-chalcone (14): $\quad$ To a solution of 4-hydroxy-chalcone $\mathrm{E}_{4}(0.5 \mathrm{~g}, 2.11 \mathrm{mmol})$ in dry acetone was added dried $\mathrm{K}_{2} \mathrm{CO}_{3}$ (4eq.) and 2-chloroethyl-piperidine $(0.32 \mathrm{~g}$, $2.35 \mathrm{mmol})$. This reaction mixture was refluxed for $8-9 \mathrm{hrs}$ at $70^{\circ} \mathrm{C}$ to get 27. Solid, Yield: $84.10 \%$, m. p.: $105-28^{\circ} \mathrm{C}$, MS (ESI): $350(\mathrm{M}+1)$, IR (KBr): 3021, 1602, 64, ${ }^{1} \mathrm{HNMR}\left(300 \mathrm{MHz}, \mathrm{CDCl}_{3}\right)$ ), $\delta 8.04$ (d, J = 8.76 $\mathrm{Hz}, 2 \mathrm{H}, 2$, 6'-H), $7.80(\mathrm{~d}, \mathrm{~J}=15.63 \mathrm{~Hz}, 1 \mathrm{H}, \beta-\mathrm{H}), 7.56(\mathrm{~d}, \mathrm{~J}=8.49 \mathrm{~Hz}$, $2 \mathrm{H}, 2,6-\mathrm{H}), 7.52(\mathrm{~d}, \mathrm{~J}=16.68 \mathrm{~Hz}, 1 \mathrm{H}, \alpha-\mathrm{H}), 7.24(\mathrm{~d}, \mathrm{~J}=7.6 \mathrm{~Hz}, 2 \mathrm{H}, 3$, 5- H), $7.00\left(\mathrm{~d}, \mathrm{~J}=8.76 \mathrm{~Hz}, 2 \mathrm{H}, 3^{\prime}, 5^{\prime}-\mathrm{H}\right), 4.24\left(\mathrm{t}, 2 \mathrm{H}, \mathrm{OCH}_{2}\right), 2.87(\mathrm{t}, 2 \mathrm{H}$, $\left.\mathrm{NCH}_{2}\right), 2.60\left(\mathrm{~s}, 4 \mathrm{H}, \mathrm{NCH}_{2}\right), 2.41\left(\mathrm{~s}, 3 \mathrm{H}, \mathrm{CH}_{3}\right), 1.69-1.27\left(\mathrm{~m}, 6 \mathrm{H}, \mathrm{CH}_{2}\right)$.

4'-(2-Azepan-1-yl-ethoxy)-4-methyl-chalcone (15): To a solution of 4-hydroxy-chalcone $\mathrm{E}_{4}(0.5 \mathrm{~g}, 2.11 \mathrm{mmol})$ in dry acetone was added dried $\mathrm{K}_{2} \mathrm{CO}_{3}$ (5eq.) and 2-chloroethyl-azepane (0.35 g, $2.35 \mathrm{mmol}$ ). This reaction mixture was refluxed for $8-9 \mathrm{hrs}$ at $70^{\circ} \mathrm{C}$ to get 28 . Solid, 
Yield: $78.74 \%$, m. p.: $12-14^{\circ} \mathrm{C}$, MS (ESI): $364(\mathrm{M}+1)$, IR (KBr): 3422, 473, 761, ${ }^{1} \mathrm{HNMR}\left(300 \mathrm{MHz}, \mathrm{CDCl}_{3}\right), \delta 8.04(\mathrm{~d}, \mathrm{~J}=8.85 \mathrm{~Hz}, 2 \mathrm{H}, 2$ ', 6'$\mathrm{H}), 7.80(\mathrm{~d}, \mathrm{~J}=15.60 \mathrm{~Hz}, 1 \mathrm{H}, \beta-\mathrm{H}) 7.56(\mathrm{~d}, \mathrm{~J}=8.34 \mathrm{~Hz}, 2 \mathrm{H}, 2,6-\mathrm{H}), 7.52$ $(\mathrm{d}, \mathrm{J}=15.81 \mathrm{~Hz}, 1 \mathrm{H}, \alpha-\mathrm{H}), 7.24(\mathrm{~d}, \mathrm{~J}=7.9 \mathrm{~Hz}, 2 \mathrm{H}, 3,5-\mathrm{H}), 7.00(\mathrm{~d}, \mathrm{~J}=$ $\left.8.85 \mathrm{~Hz}, 2 \mathrm{H}, 3,5^{\prime}-\mathrm{H}\right), 4.18\left(\mathrm{t}, 2 \mathrm{H}, \mathrm{OCH}_{2}\right), 3.01\left(\mathrm{t}, 2 \mathrm{H}, \mathrm{NCH}_{2}\right), 2.82(\mathrm{~m}$, $\left.4 \mathrm{H}, \mathrm{NCH}_{2}\right), 2.41\left(\mathrm{~s}, 3 \mathrm{H}, \mathrm{CH}_{3}\right), 1.69-1.27\left(\mathrm{~m}, 8 \mathrm{H}, \mathrm{CH}_{2}\right)$.

4'-(2-Diethylamino-ethoxy)-4-nitro-chalcone (16): To a solution of 4-hydroxy-chalcone $\mathrm{E}_{5}(0.5 \mathrm{~g}, 1.85 \mathrm{mmol})$ in dry acetone was added dried $\mathrm{K}_{2} \mathrm{CO}_{3}$ (3eq.) and 2-chloroethyl-diethylamine (0.26 g, 2.32 $\mathrm{mmol}$ ). This reaction mixture was refluxed for $8-9 \mathrm{hrs}$ at $70^{\circ} \mathrm{C}$ to get 29 . Solid, Yield: $84.21 \%$, m. p.: $32-34^{\circ} \mathrm{C}$, MS (ESI): $369(\mathrm{M}+1)$, IR (KBr): 3440, 1660, 809, ${ }^{1} \mathrm{HNMR}\left(200 \mathrm{MHz}, \mathrm{CDCl}_{3}\right), \delta 8.28(\mathrm{~d}, \mathrm{~J}=8.76 \mathrm{~Hz}$, $2 \mathrm{H}, 3,5-\mathrm{H}), 8.04\left(\mathrm{~d}, \mathrm{~J}=8.84 \mathrm{~Hz}, 2 \mathrm{H} 2^{\prime}, 6^{\prime}-\mathrm{H}\right), 7.82(\mathrm{~d}, \mathrm{~J}=15.84 \mathrm{~Hz}, 1 \mathrm{H}$, $\beta-\mathrm{H}), 7.79(\mathrm{~d}, \mathrm{~J}=8.67 \mathrm{~Hz}, 2 \mathrm{H}, 2,6-\mathrm{H}), 7.27(\mathrm{~d}, \mathrm{~J}=15.68 \mathrm{~Hz}, 1 \mathrm{H}, \alpha-\mathrm{H})$, $7.01(\mathrm{~d}, \mathrm{~J}=8.86 \mathrm{~Hz}, 2 \mathrm{H}, 3,5$ ' $-\mathrm{H}), 4.14\left(\mathrm{t}, 2 \mathrm{H}, \mathrm{OCH}_{2}\right), 2.8\left(\mathrm{t}, 2 \mathrm{H}, \mathrm{NCH}_{2}\right.$ ), $2.66\left(\mathrm{q}, 4 \mathrm{H},-\mathrm{NCH}_{2}\right), 1.09\left(\mathrm{t}, 6 \mathrm{H},-\mathrm{CH}_{3}\right)$.

4'-(2-Piperidin-1-yl-ethoxy)-4-nitro-chalcone (17): To a solution of 4-hydroxy-chalcone $\mathrm{E}_{5}(0.5 \mathrm{~g}, 1.85 \mathrm{mmol})$ in dry acetone was added dried $\mathrm{K}_{2} \mathrm{CO}_{3}$ (5eq.) and 2-chloroethyl-piperidine $(0.28 \mathrm{~g}, 2.09 \mathrm{mmol})$. This reaction mixture was refluxed for $8-9 \mathrm{hrs}$ at $70^{\circ} \mathrm{C}$ to get 30 . Solid, Yield: $83.80 \%$, m. p.: $42^{\circ} \mathrm{C}$, MS (ESI): $381(\mathrm{M}+1)$, IR (KBr): 211,1753 , $74,65,{ }^{1} \mathrm{HNMR}\left(300 \mathrm{MHz}, \mathrm{CDCl}_{3}\right), \delta 8.29(\mathrm{~d}, \mathrm{~J}=8.76 \mathrm{~Hz}, 2 \mathrm{H}, 3,5-\mathrm{H})$, $8.06(\mathrm{~d}, \mathrm{~J}=8.85 \mathrm{~Hz}, 2 \mathrm{H}$ 2', 6'-H) $7.83(\mathrm{~d}, \mathrm{~J}=15.69 \mathrm{~Hz}, 1 \mathrm{H}, \beta-\mathrm{H}), 7.80(\mathrm{~d}$, $\mathrm{J}=8.70 \mathrm{~Hz}, 2 \mathrm{H}, 2,6-\mathrm{H}), 7.67(\mathrm{~d}, \mathrm{~J}=15.5 \mathrm{~Hz}, 1 \mathrm{H}, \alpha-\mathrm{H}), 7.03(\mathrm{~d}, \mathrm{~J}=8.85$ $\mathrm{Hz}, 2 \mathrm{H}, 3,5$ '- $\mathrm{H}), 4.22\left(\mathrm{t}, 2 \mathrm{H}, \mathrm{OCH}_{2}\right), 2.83\left(\mathrm{t}, 2 \mathrm{H}, \mathrm{NCH}_{2}\right), 2.5423(\mathrm{~m}$, $\left.4 \mathrm{H}, \mathrm{NCH}_{2}\right), 1.67-1.60\left(\mathrm{~m}, 6 \mathrm{H}, \mathrm{CH}_{2}\right)$.

4'-(2-Azepan-1-yl-ethoxy)-4-nitro-chalcone (18): To a solution of 4-hydroxy-chalcone $\mathrm{E}_{5}(0.5 \mathrm{~g}, 1.85 \mathrm{mmol})$ in dry acetone was added dried $\mathrm{K}_{2} \mathrm{CO}_{3}$ (4eq.) and 2-chloroethyl-azepane $(0.31 \mathrm{~g}, 2.26 \mathrm{mmol})$. This reaction mixture was refluxed for $8-9 \mathrm{hrs}$ at $70^{\circ} \mathrm{C}$ to get 31 . Solid, Yield: $82.19 \%$, m. p.: $80-83^{\circ} \mathrm{C}$, MS (ESI): $312(\mathrm{M}+1)$, IR (KBr): 3021, 1600, 64, ${ }^{1} \mathrm{HNMR}\left(200 \mathrm{MHz}, \mathrm{CDCl}_{3}\right), \delta 8.28(\mathrm{~d}, \mathrm{~J}=8.76 \mathrm{~Hz}, 2 \mathrm{H}, 3,5-\mathrm{H}), 8.04(\mathrm{~d}$, $\left.\mathrm{J}=8.82 \mathrm{~Hz}, 2 \mathrm{H} 2^{\prime}, 6^{\prime}-\mathrm{H}\right) 7.81(\mathrm{~d}, \mathrm{~J}=15.84 \mathrm{~Hz}, 1 \mathrm{H}, \beta-\mathrm{H}), 7.78(\mathrm{~d}, \mathrm{~J}=8.68$ $\mathrm{Hz}, 2 \mathrm{H}, 2,6-\mathrm{H}), 7.64(\mathrm{~d}, \mathrm{~J}=15.7 \mathrm{~Hz}, 1 \mathrm{H}, \alpha-\mathrm{H}), 7.01(\mathrm{~d}, \mathrm{~J}=8.82 \mathrm{~Hz}, 2 \mathrm{H}$, 3', 5'-H), $4.19\left(\mathrm{t}, 2 \mathrm{H}, \mathrm{OCH}_{2}\right), 3.02\left(\mathrm{t}, 2 \mathrm{H}, \mathrm{NCH}_{2}\right), 2.83-2.80(\mathrm{~m}, 4 \mathrm{H}$, $\left.\mathrm{NCH}_{2}\right), 1.64\left(\mathrm{~s}, 8 \mathrm{H}, \mathrm{CH}_{2}\right)$.

4'-(2-Diethylamino-ethoxy)-2-chloro-chalcone (19): To a solution of 4-hydroxy chalcone $\mathrm{E}_{6}(0.5 \mathrm{~g}, 1.27 \mathrm{mmol})$ in dry acetone was added dried $\mathrm{K}_{2} \mathrm{CO}_{3}$ (3eq.) and 2-chloroethyl-diethylamine $(0.27 \mathrm{~g}, 2.40$ $\mathrm{mmol})$. This reaction mixture was refluxed for $8-9 \mathrm{hrs}$ at $70^{\circ} \mathrm{C}$ to get 32 . Liquid, Yield: 78.32\%, MS (ESI): 358 (M+1), IR (Neat): 2139, 479, 761, ${ }^{1} \mathrm{HNMR}\left(300 \mathrm{MHz}, \mathrm{CDCl}_{3}\right), \delta 8.15(\mathrm{~d}, \mathrm{~J}=15.5 \mathrm{~Hz}, 1 \mathrm{H}, \beta-\mathrm{H}), 8.02(\mathrm{~d}$, $\mathrm{J}=8.85 \mathrm{~Hz}, 2 \mathrm{H} \mathrm{2}$ ', 6'-H), 7.76-7.73 (m, 1H, 3-H), $7.49(\mathrm{~d}, \mathrm{~J}=15.5 \mathrm{~Hz}$, $1 \mathrm{H}, \mathrm{\alpha}-\mathrm{H}), 7.42(\mathrm{~m}, 1 \mathrm{H}, 6-\mathrm{H}), 7.33-7.30(\mathrm{~m}, 2 \mathrm{H}, 4,5-\mathrm{H}), 6.16(\mathrm{~d}, \mathrm{~J}=8.85$ $\left.\mathrm{Hz}, 2 \mathrm{H}, 3^{\prime}, 5^{\prime}-\mathrm{H}\right), 4.13\left(\mathrm{t}, 2 \mathrm{H}, \mathrm{OCH}_{2}\right), 2.7\left(\mathrm{t}, 2 \mathrm{H},-\mathrm{CH}_{2} \mathrm{~N}\right), 2.64(\mathrm{q}, 4 \mathrm{H}$, $\left.-\mathrm{NCH}_{2}\right), 1.07\left(\mathrm{t}, 6 \mathrm{H},-\mathrm{CH}_{3}\right)$.

4'-(2-Piperidin-1-yl-ethoxy)-4-methoxy-chalcone (20): To a solution of 4-hydroxy-chalcone $\mathrm{E}_{7}(0.5 \mathrm{~g}, 1.13 \mathrm{mmol})$ in dry acetone was added dried $\mathrm{K}_{2} \mathrm{CO}_{3}$ (4eq.) and 2-chloroethyl-piperidine (0.3 g, 2.19 $\mathrm{mmol})$. This reaction mixture was refluxed for $8-9 \mathrm{hrs}$ at $70^{\circ} \mathrm{C}$ to get 33. Liquid, Yield: 75.34\%, MS (ESI): $366(\mathrm{M}+1)$, IR (Neat): 3418, 478, ${ }^{1} \mathrm{HNMR}\left(300 \mathrm{MHz}, \mathrm{CDCl}_{3}\right), \delta 7.80(\mathrm{~d}, \mathrm{~J}=15.66 \mathrm{~Hz}, 1 \mathrm{H}, \beta-\mathrm{H}), 7.62(\mathrm{~d}$, $\left.\mathrm{J}=8.64 \mathrm{~Hz}, 2 \mathrm{H} 2^{\prime}, 6^{\prime}-\mathrm{H}\right), 7.40(\mathrm{~d}, \mathrm{~J}=15.63 \mathrm{~Hz}, 1 \mathrm{H}, \alpha-\mathrm{H}), 7.59-7.56(\mathrm{~m}$, 2H, 4, 6-H), 7.41 (s, 1H, 2-H), $7.14(\mathrm{dd}, \mathrm{J}=2.1,8.07 \mathrm{~Hz}, 1 \mathrm{H}, 5-\mathrm{H}), 6.13$ (d, J = 8.76, 2H, 3', 5'-H), $4.20\left(\mathrm{t}, 2 \mathrm{H}, \mathrm{OCH}_{2}\right), 3.88\left(\mathrm{~s}, 3 \mathrm{H}, \mathrm{OCH}_{3}\right), 2.82$ $\left(\mathrm{t}, 2 \mathrm{H}, \mathrm{CH}_{2} \mathrm{~N}\right), 2.55\left(\mathrm{~m}, 4 \mathrm{H}, \mathrm{NCH}_{2}\right), 1.27-1.60\left(\mathrm{~m}, 4 \mathrm{H}, \mathrm{CH}_{2}\right), 1.51-1.45$ (m, $2 \mathrm{H}, \mathrm{CH}_{2}$ ).

\section{Biology}

Materials: Streptozotocin and metformin were obtained from Sigma Chemical Company (St. Louis, USA). All the chemicals and reagents used were of analytical grade. Glucostrips were purchased from Roche Diagnostics, Germany.

Animals: 10 to 12 weeks old male albino rats of Sprague Dawley strain weighing around $140 \pm 20 \mathrm{gm}$, procured from National Laboratory Animal Centre of CSIR-Central Drug Research Institute [CDRI], Lucknow, India. All procedures on these animals were conducted in strict accordance with the care and use of laboratory animals after getting the necessary approval by Institutional Animal Ethical Committee and were conducted in accordance with the guidelines of the Committee for the Purpose of Control and Supervision of Experiments on Animals (CPCSEA) formed by the Government of India in 1964. Animals were always placed in polypropylene cages in groups of five to six. The following norms were always followed for animal room environment: Temperature $23 \pm 2^{\circ} \mathrm{C}$ : Humidity 50-60\%: Light 300 Lux at floor level with regular $12 \mathrm{hr}$ light cycle. The animals had always access to pellet diet and tap water unless stated otherwise.

Improvement on glucose tolerance in normal rats (SLM): The basal blood glucose level of each animal was measured by glucometer (Accuchek) after an overnight starvation. Animals showing fasting blood glucose level between 60 to $80 \mathrm{mg} / \mathrm{dl}$ were selected and divided into groups of five animals in each group. Rats of test groups were orally administered the suspension of the test sample prepared in $1.0 \%$ gum acacia at the dose of $100 \mathrm{mg} / \mathrm{kg}$ b.wt. and standard drug metformin at the dose of $100 \mathrm{mg} / \mathrm{kg}$ body wt. Animals of control group were given an equal amount of $1.0 \%$ gum acacia and termed as sham control. Oral loading of sucrose (10 g/kg b.wt.) was given to each animal after $30 \mathrm{~min}$ post administration of the test sample/vehicle. Blood glucose of each animal was again determined from the tail vein at 30,60, 90 and 120 min post sucrose load by gluco-meter. Food but not water was withheld from the cages during the period of experimentation. Comparing the AUC of treated group with that of sham treated control group determined the percentage improvement on oral glucose tolerance post sucrose load by the test substance.

Decline in blood glucose level of Streptozotocin-induced diabetic rats (STZ): The fresh solution of STZ [60 mg/kg] in 100 $\mathrm{mM}$ citrate buffer, $\mathrm{pH} 4.5$ was prepared and calculated amount of the fresh solution was immediately injected to overnight fasted rats intraperitoneally. Two days later baseline blood glucose was drawn from tail vein and glucose levels above $300 \mathrm{mg} / \mathrm{dl}$ were taken for the study and determined by glucostrips to confirm the induction of diabetes. Rats having hyperglycemia of the range of $300-400 \mathrm{mg} / \mathrm{dl}$ were considered diabetic, selected and were divided into groups of five animals each. One Group were used for normal control receives only vehicle [gum-acacia] and this group was termed as sham control. Other groups received the test samples and standard antidiabetic drugs at $100 \mathrm{mg} / \mathrm{kg}$ dose. The blood glucose was measured in blood sample obtained from tail vein at $30,60,90,120,180,240,300$ and 1440 min post administration of test sample/vehicle. Food but not water was withheld from the cages upto $300 \mathrm{~min}$ period of experiment. The animals were allowed to feed over night to overcome drug induced hypoglycaemia after $300 \mathrm{~min}$. The \% decline in AUC of test samples or standard antidiabetic drug treated groups determined the $\%$ blood glucose lowering activity.

Statistical analysis: With the help of Area under curve (AUC) method (Prism Software), the quantitative glucose tolerance of every animal was calculated. The standard fall in AUC of experimental group compared to control group was always termed as\% enhancement 
on glucose tolerance or anti-hyperglycemic activity. The results are expressed as mean \pm SEM and statistical study was carried out to determine the level of significance by one-way ANOVA followed by Dunnett's test by graph pad prism software package. It is denoted by $\mathrm{p}$ values. Statistically significant differences were put at following levels * represents $\mathrm{p}<0.05,{ }^{* *}$ represents $\mathrm{p}<0.01$.

\section{Quantitative structure activity relationship}

The calibration set and the prediction set: Chalcone based Aryloxyethylamines as Antihyperglycemic agents (Table 1) were included in the calibration set. The Antihyperglycemic activity was expressed by means of the equation $A=-\log$ (activity). In this mentioned study it has been also observed that A series of chalcone aryloxyethylamines of type 1-16 (Table 1) act as their anti-hyperglycemic activity, with variable efficacies, depending on their chemical structure. The prediction set contains 13 other not yet synthesized substituted of chalcone aryloxyethylamines, having unknown observed values of activity (Table 1) and structure presented in Figure 2. The discovery of novel bioactive molecule is the primary goal of computational drug discovery. The discovery of novel bioactive molecule is the primary goal of computational drug discovery. The virtual constructions of the molecules and geometry optimization have been done using the Molecular Mechanics force field $\mathrm{MM}^{+}$of Hyper Chem software [17] (version 7.52, HyperCube Inc. Florida USA). The MM+ force field is an extension of latest MM2 force field which was developed by Allinger and co-workers [18,19]. Separately, for each molecule, 3224DRAGON [20] (version 5.5, Talete srl, Milano, Italy) descriptors have been calculated. Several criteria were used to reduce the DRAGON descriptors while optimizing the information content of the descriptors set. First, descriptors for which no value was available for all the compounds were disregarded. Second, descriptors of which the value is constant (or nearconstant) inside each group of descriptors were excluded. After these automatic screening procedures, a set of 1598 DRAGON descriptors was obtained for further analysis. The statistical calculations used for obtaining the QSAR equations were done with PRECLAV as reported earlier [21-23].

Descriptor calculation and quality of the model: The program PRECLAV (8.) computes type (2) multilinear QSARs.

$$
\mathrm{A}=\mathrm{C}_{0}+\Sigma \mathrm{C}_{\mathrm{i}} \cdot \mathrm{D}_{\mathrm{i}}
$$

$\mathrm{i}=1$

where, $\mathrm{A}$ is (the value of) activity; $\mathrm{C}_{0}$ is the free term intercept); $\mathrm{C}_{\mathrm{i}}$ are coefficients (weighting factors); $\mathrm{D}_{\mathrm{i}}$ are (the value of) significant descriptors; $k$ is the number of descriptors.

The square of Pearson linear correlation $r^{2}$ of observed/computed values, the Fisher function $F$, the standard error of estimation SEE, and the quality function $Q^{1}$ are criteria for the quality of prediction for the molecules in calibration set.

$$
\begin{aligned}
& \mathrm{F}=\mathrm{r}^{2} /\left(1-\mathrm{r}^{2}\right) \cdot(\mathrm{N}-p) / p \\
& \mathrm{SEE}=\left[\left(\Sigma \Delta^{2}\right) /(\mathrm{N}-1)\right]^{1 / 2} \\
& \mathrm{Q}=\mathrm{r}^{2} \cdot(1-p / \mathrm{N})
\end{aligned}
$$

where: $p$ is number of descriptors; $\mathrm{N}$ is number of molecules in the calibration set; $\Delta$ is difference $A_{\text {obs }}-A_{\text {calc }}$. The descriptors included in the best (by $Q$ function) QSAR are named 'predictors'. The relative utility of predictors is computed by the formula (6).

$$
\mathrm{U}=\left(\mathrm{R}^{2}-\mathrm{r}^{2}\right) /\left(1-\mathrm{r}^{2}\right)
$$

where $\mathrm{R}^{2}$ is the square of Pearson correlation between the observed values and the computed values (using $p$ predictors) $r^{2}$ is the square of Pearson correlation between the observed values and the calculated values (using the $p$ - 1 predictors, i.e., the QSAR equation without the analyzed predictor)

After computation of $U^{24}$ for each predictor, the values of $U$ are normalized by the highest of them (the highest value for $U$ becomes $1000)$. The predictors with high enough value of $U(U>500)$ can be considered 'with high relative utility'. These predictors are useful because they correlate well with $\mathrm{A}_{\mathrm{obs}}$ values and present low correlation with other descriptors.

PRECLAV [24] calculates square of cross-validated correlation $\mathrm{r}_{\mathrm{CV}}^{2}$ using LHO (Leave Half Out) method. However, this usual method is applied after ordering of molecules in calibration set according to the observed values of activity. Therefore, the cross-validated function $\mathrm{r}_{\mathrm{CV}}^{2}$ is a measure of homogeneity of calibration set from the point of view of predictors' set, i.e., from the point of view of structure-activity relationship. A low value $(<0.4)$ of $\mathrm{r}_{\mathrm{CV}}^{2}$ means 'the QSAR for molecules having high values of activity and the QSAR for molecules having low values of activity include the same descriptors, but very different weighting factors'. Actually, the computation of $\mathrm{r}_{\mathrm{CV}}{ }$ is a very drastic 'internal validation test'.

Applicability of domain and detection of outliers: A QSAR model can be used for showing new compounds if its domain of application is defined $[25,26]$. The need to characterize the model applicability domain is also reflected in the OECD guiding principle for QSAR model validation $[27,28]$. QSAR model should only be used for making predictions of compounds fall within the specified domain may be considered reliable. Extent of extrapolation [29,30] is one simple approach to define the applicability of the domain. It is based on the calculation of the hat diagonal (leverage) $h_{i}$ for each chemical, where the QSAR model is used to predict its activity.

$$
\mathbf{h}_{\mathrm{i}}=\mathbf{1} / 4 \mathbf{X}_{\mathrm{i}}^{\mathrm{T}}\left(\mathbf{X}^{\mathrm{T}} \mathbf{X}\right)^{-1} \mathbf{X}_{\mathrm{i}}
$$

In equation $9, \mathrm{x}_{\mathrm{i}}$ is the descriptor-row vector of the query molecule and $\mathrm{X}$ is the $\mathrm{kx} \mathrm{n}$ matrix containing the $\mathrm{k}$ descriptor values for each one of the $\mathrm{n}$ training molecules. A hat diagonal (leverage) value $>3(\mathrm{k}+1) / \mathrm{n}$ leverage warning limit is considered large.

Outliers are compounds that are poorly fit by the regression model. Outlying compounds should not be removed unless a good reason for their removal can be given. The variance of the observed residuals is not constant. This makes comparisons among the residuals difficult. One solution is to standardize the residuals $[31,32]$, by dividing by their standard deviations. This gives a set of standardized residuals. The cross-validated LOO standardized residuals is a $\mid$ RStudent $\mid$ that has the impact of a single observation removed from the mean square error. A molecule is defined as an outlier in which $\mid$ RStudent $\mid>2$ [32-34].

To visualize the applicability of domain of a developed QSAR model, William plot was used. In the William plot, $\mid$ RStudent $\mid$ versus leverage values $\left(h_{i}\right)$ are plotted. This plot could be used for an immediate and simple graphical detection of both the response outliers and structurally influential compounds in a model. It must be noted that compounds with high value of leverage and good fitting in the developed model can stabilize the model. On the other hand, compounds with bad fitting in the developed model may be outliers. Thus, combination of leverage and the $\mid$ RStudent $\mid$ could be used for assigning the applicability of domain [35-37].

\section{Results and Discussion}

The statistical computations were conducted using the specific 
Citation: Shukla P, Pratap R, Singh S, Rahuja N, Prasad AK, et al. (2017) In Silco Molecular Modeling of Chalcone Based Aryloxyethylamines as Antihyperglycemic Agents. Med Chem (Los Angeles) 7: 226-234. doi: 10.4172/2161-0444.1000461

formulas and procedures of PRECLAV program algorithm. Using only the "significant" descriptors, PRECLAV computes ten thousand QSAR type (3) multilinear equations. The quality of the obtained equation is reflected by the value of the $Q$ function and also by values of some usual statistical functions. During the PRECLAV MLR analysis using Dragon descriptor, we observed that the 3-parametric model has the highest value of the $Q$ function for anti-hyperglycemic activity and also has the highest predictive power as follows:

Dependent property: antihyperglycemic activity $(A)$

Molecules number in calibration set: 16

Number of "significant" descriptors in presence of set: 277

$A=4.3733-1.0262$ (Eig06_A) + 6.3981 (G2p) -7.057 (G2m)

Eig06_A = eigen value n. 6 from augmented edge adjacency mat. weighted by edge degree Edge adjacency indices Eigenvalues $(\mathrm{U}=1000)$

$\mathrm{G} 2 \mathrm{p}=2$ nd component symmetry directional WHIM index / weighted by polarizability $(\mathrm{U}=\mathbf{8 2 9})$

$\mathrm{G} 2 \mathrm{~m}=2 \mathrm{nd}$ component symmetry directional WHIM index / weighted by mass WHIM descriptors Directional descriptors $(U=912)$, $\mathrm{SEE}=0.0466 ; r 2=0.925 ; F=53.4748 ; r 2, c v=0.8777, \mathrm{rpred}^{2}: 0.85599$. $\mathrm{SEE}=$ Standard Error of Estimation, $r 2=$ Pearson square correlation, $F=$ Fisher function, $r 2 \mathrm{cv}=$ Pearson cross validated square correlation (Leave one out method), rpred $^{2}=$ predictive $\mathrm{r} 2$ According to algebraic sign of coefficients in QSAR formula and the value of utility $U$ the main factor in influence on activity value is the Eigenvalues [Eigenvalues of the edge adjacency matrix (Eigk_EA), augmented edge adjacency matrices (Eigk_AEA(w) and unsymmetrical weighted edge adjacency matrices (Eigk_EA(w)) are also provided by Dragon and Directional WHIM descriptors [WHIM descriptors (Weighted Holistic Invariant Molecular descriptors) are geometrical descriptors based on statistical indices calculated on the projections of the atoms along principal axes.

WHIM descriptors are built in such a way as to capture relevant molecular 3D information regarding molecular size, shape, symmetry and atom distribution with respect to invariant reference frames)] Play dominant role in activity. The negative correlation of Eig06A shows that, increases this descriptor value decreases the activity and positive correlation of the G2p shows the increase the polarizibitly increase the activity.

\section{Validation of the computation procedure}

For the validation of the method, we have proceeded to a QSAR study with a validation set and reduced calibration set. The set in bold letter with star containing compound in Table 1 are the most significant is the correlation between the calculated and selected for validation set (comp. no 2,10,12 and 16). The selection of the validation set should be such that it captures all the features and characteristics of the whole set of molecules validation set was extracted from the homogenized calibration. From the point of view of them considerations discussed here, the experimental values of activity for the molecules in the validation set. In Table 2 and Figure 1 there are listed the calculated values and the experimental values of the anti-hyperglycemic activity power for the molecules in the validation set. We have found good predictive $\mathrm{r}^{2}$.

Applicability domain: We used |RStudent| of observed inhibitory activity calculated by the obtained models and hat diagonal (leverage) for assigning applicability of domain (AD). Values for leverage have been calculated for both calibration set and prediction set compounds showing in Table 2. Applicability of domain for the developed model

\begin{tabular}{|c|c|c|c|c|c|c|c|}
\hline $\begin{array}{c}\text { Comp. } \\
\text { No. }\end{array}$ & (A) Obs. & (A)Est. & Res. & $\begin{array}{c}\mathbf{R} \\
\text { Student }\end{array}$ & Diagonal & $\begin{array}{c}\text { Comp. } \\
\text { No. }\end{array}$ & $\begin{array}{c}\text { A (predicted } \\
\text { Value) }\end{array}$ \\
\hline 3 & 1.2455 & 1.193 & 0.053 & 1.0871 & 0.1133 & $\mathrm{~A}$ & 3.001 \\
\hline $5^{*}$ & 1.1461 & 1.12 & 0.026 & 0.5578 & 0.2245 & $\mathrm{~B}$ & 2.683 \\
\hline 7 & 1.2553 & 1.305 & -0.05 & -1.031 & 0.1304 & $\mathrm{C}$ & 2.734 \\
\hline 8 & 0.8162 & 0.858 & -0.042 & -1.0729 & 0.428 & $\mathrm{D}$ & 2.765 \\
\hline 9 & 0.9872 & 0.952 & 0.035 & 0.8383 & 0.3809 & $\mathrm{E}$ & 2.422 \\
\hline 10 & 1.0864 & 1.136 & -0.049 & -1.1263 & 0.2783 & $\mathrm{~F}$ & 2.53 \\
\hline 11 & 1.3096 & 1.357 & -0.047 & -1.0226 & 0.2083 & $\mathrm{G}$ & 2.045 \\
\hline 12 & 1.3655 & 1.288 & 0.078 & 1.669 & 0.0845 & $\mathrm{H}$ & 2.692 \\
\hline 13 & 1.2041 & 1.239 & -0.035 & -0.6915 & 0.0973 & $\mathrm{I}$ & 2.052 \\
\hline $14^{*}$ & 1.3636 & 1.3 & 0.063 & 1.3873 & 0.1713 & $\mathrm{~J}$ & 2.689 \\
\hline 15 & 1.3541 & 1.397 & -0.043 & -0.9113 & 0.1776 & $\mathrm{~K}$ & 2.283 \\
\hline $16^{*}$ & 1.1553 & 1.113 & 0.042 & 0.8367 & 0.0923 & $\mathrm{~L}$ & 2.395 \\
\hline 17 & 1.3979 & 1.372 & 0.026 & 0.8189 & 0.6436 & $\mathrm{M}$ & 1.835 \\
\hline 18 & 1.1206 & 1.178 & -0.058 & -1.2749 & 0.205 & - & - \\
\hline 19 & 1.3979 & 1.395 & 0.003 & 0.0707 & 0.2451 & - & - \\
\hline $20^{*}$ & 0.9886 & 0.99 & -0.002 & -0.0437 & 0.5196 & - & - \\
\hline
\end{tabular}

Table 2: Observed anti-hyperglycemic activity and their corresponding $A$ value where $A=-\log$, anti-hyperglycemic activity, estimated inhibition constant $(A)$, hat diagonal, standardized residual, $|R S t u d e n t|$ of the calibration set molecules of chalcone having good \% lowering in STZ $(24 \mathrm{hrs})$ model and predicted value of A-M not yet synthesized compounds.
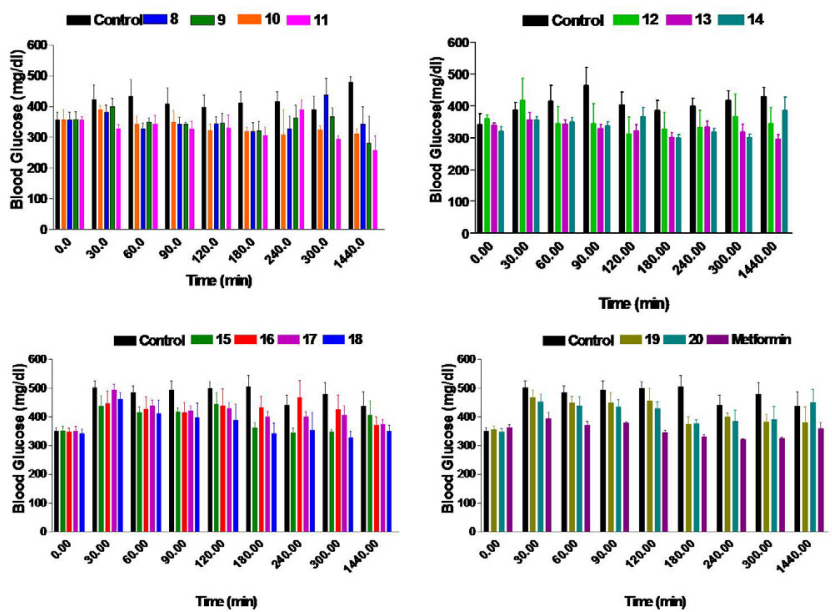

Figure 3: Effect of synthetic compounds and standard antidiabetic drug metformin on blood glucose profile of streptozotocin-induced diabetic rats. Values are mean \% change in 5 animals of a group; significance: ${ }^{*} p<0.05$, ${ }^{* *} p<0.01$ when compared with control.
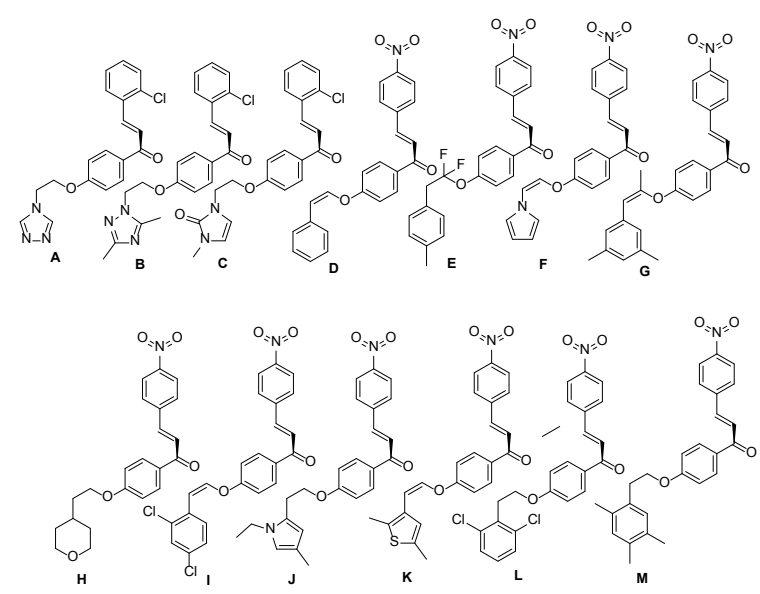

Figure 4: Structure of compounds A-M predicted as antihyperglycemic agents 
Citation: Shukla P, Pratap R, Singh S, Rahuja N, Prasad AK, et al. (2017) In Silco Molecular Modeling of Chalcone Based Aryloxyethylamines as Antihyperglycemic Agents. Med Chem (Los Angeles) 7: 226-234. doi: 10.4172/2161-0444.1000461

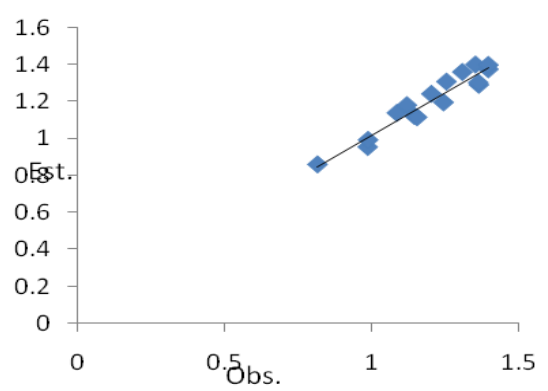

Figure 5: Observed versus estimated inhibitory activity $(A)$ of Calibration set.

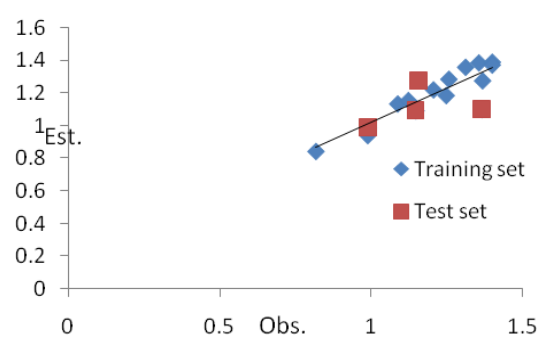

Figure 6: Observed versus estimated inhibitory activity $(A)$ of training set and test set.

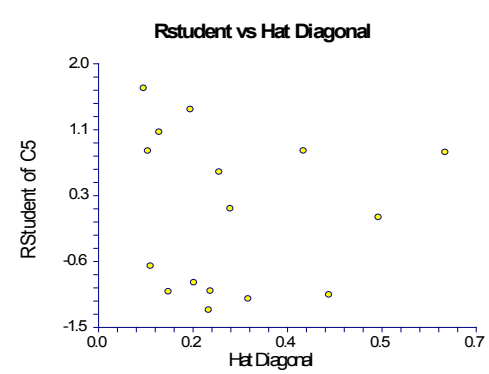

Figure 7: |RStudent| of observed vs. Hat Diagonal.

of calibration set is shown in William plot (Figures 3-7). Influential compounds are points with leverage value higher than the warning leverage limit 1 , so outliers are not present. It can be seen in the William plot; all molecules in calibration set lie in the application domain of the developed model. The computed activity of the prediction set compounds is also within limit of hat diagonal.

\section{Conclusions}

The compounds with alkylaminoethoxy group on ring-A and electron withdrawing group on ring-B exhibited potential antihyperglycemic activity in SLM and STZ models. In calibration set polarizibilty and Mass play dominant role for the activity. Many molecules in proposed prediction set have much higher computed activity than observed value and their hat diagonal within limit. Thus, attempts have been made to design and develop novel drugs against antihyperglycemic activity on a rational basis so as to decreases the test and fault issue and predict the biological activity before synthesis.

\section{Acknowledgements}

Authors are grateful to Director, CDRI, Lucknow, India, for constan encouragement of the drug development program for metabolic diseases and Sophisticated Analytical Instrumentation Facility (SAIF) group for spectral data. Poonam is grateful to UGC, New Delhi, for awarding Junior Research Fellowship and University of Delhi for other support.

\section{References}

1. Ginsberg H, Plutzky J, Sobel BE (1999) A review of metabolic and cardiovascular effects of oral antidiabetic agents: beyond glucose-level lowering. Journal of cardiovascular risk 6: 337-346.

2. Gavrilova O, Marcus-Samuels B, Graham D, Kim JK, Shulman GI, et al. (2000) Surgical implantation of adipose tissue reverses diabetes in lipoatrophic mice. Journal of Clinical Investigation 105: 271.

3. Murphy JE, Zhou S, Giese K, Williams LT, Escobedo JA et al. (1997) Longterm correction of obesity and diabetes in genetically obese mice by a single intramuscular injection of recombinant adeno-associated virus encoding mouse leptin. Proceedings of the National Academy of Sciences 94: 13921-13926.

4. Nourparvar A, Bulotta A, Di Mario U, Perfetti R (2004) Novel strategies for the pharmacological management of type 2 diabetes. Trends in pharmacological sciences 25: 86-91.

5. Arch JR, Ainsworth AT (1983) Thermogenic and antiobesity activity of a novel beta-adrenoceptor agonist (BRL 26830A) in mice and rats. The American journal of clinical nutrition 38: 549-558.

6. Holloway BR, Howe R, Rao BS, Stribling D (1992) ICI D7114: a novel selective adrenoceptor agonist of brown fat and thermogenesis. The American journal of clinical nutrition 55: 262S-264S.

7. Goldberg DE, Frishman WH (1995) b3-Adrenergic Agonism: A New Concept in Human Pharmacotherapy, Futura publishing Co. Armonk: New York.

8. Leo A, Muller M (1986) Eur. Patent EP 14023, 1985. InChem. Abstr 104: 68565.

9. Shukla P, Singh AB, Srivastava AK, Pratap R (2007) Chalcone based aryloxypropanolamines as potential antihyperglycemic agents. Bioorganic \& medicinal chemistry letters 17: 799-802.

10. Bloom JD, Dutia MD, Johnson BD, Wissner A, Burns MG, et al. (1992) Disodium (R, R)-5-[2-[[2-(3-chlorophenyl)-2-hydroxyethyl] amino] propyl]-1, 3-benzodioxole-2, 2-dicarboxylate (CL 316,243). A potent. beta--adrenergic agonist virtually specific for. beta. 3 receptors. A promising antidiabetic and antiobesity agent. Journal of medicinal chemistry 35: 3081-3084.

11. Oguchi M, Wada K, Honma H, Tanaka A, Kaneko T, et al. (2000) Molecular design, synthesis, and hypoglycemic activity of a series of thiazolidine-2. 4-diones. Journal of medicinal chemistry 43: 3052-3066.

12. Monnier VM (1989) Toward a Maillard reaction theory of aging. Progress in clinical and biological research 304: 1-22.

13. Vaya J, Belinky PA, Aviram M (1997) Antioxidant constituents from licorice roots: isolation, structure elucidation and antioxidative capacity toward LDL oxidation. Free Radical Biology and Medicine 23: 302-313.

14. Chen M, Christensen SB, Zhai L, Rasmussen MH, Theander TG, et al. (1997) The novel oxygenated chalcone, 2, 4-dimethoxy-4'-butoxychalcone, exhibits potent activity against human malaria parasite Plasmodium falciparum in vitro and rodent parasites Plasmodium berghei and Plasmodium yoelii in vivo. Journal of Infectious Diseases 176: 1327-1333.

15. Nielsen SF, Christensen SB, Cruciani G, Kharazmi A, Liljefors T (1998) Antileishmanial chalcones: Statistical design, synthesis, and three-dimensional quantitative structure- activity relationship analysis. Journal of Medicinal Chemistry 41: 4819-4832.

16. Hsieh HK, Lee TH, Wang JP, Wang JJ, Lin CN (1998) Synthesis and antiinflammatory effect of chalcones and related compounds. Pharmaceutical research 15: 39-46.

17. Kumar SK, Hager E, Pettit C, Gurulingappa H, Davidson NE, et al. (2003) Design, synthesis, and evaluation of novel boronic-chalcone derivatives as antitumor agents. Journal of medicinal chemistry 46: 2813-2815.

18. Shukla P, Singh AB, Srivastava AK, Pratap R (2007) Chalcone based aryloxypropanolamines as potential antihyperglycemic agents. Bioorganic \& medicinal chemistry letters 17: 799-802.

19. Satyanarayana M, Tiwari $P$, Tripathi BK, Srivastava AK, Pratap $R$ (2004) Synthesis and antihyperglycemic activity of chalcone based aryloxypropanolamines. Bioorganic \& medicinal chemistry 12: 883-889.

20. Shukla P, Singh AB, Srivastava AK, Pratap R (2007) Chalcone based aryloxypropanolamines as potential antihyperglycemic agents. Bioorganic \& medicinal chemistry letters $17: 799-802$

21. Hyper Chem Software7.52 (2006) Hyper Cube Inc. Florida Science and Technology Park, 1115, NW. $4^{\text {th }}$ street Gainesvilla Florida, 326001, USA. 
Citation: Shukla P, Pratap R, Singh S, Rahuja N, Prasad AK, et al. (2017) In Silco Molecular Modeling of Chalcone Based Aryloxyethylamines as Antihyperglycemic Agents. Med Chem (Los Angeles) 7: 226-234. doi: 10.4172/2161-0444.1000461

22. Allinger NL (1977) Conformational analysis. 130. MM2. A hydrocarbon force field utilizing V1 and V2 torsional terms. Journal of the American Chemical Society 99: 8127-8134.

23. Allinger NL, Yuh YH (1982) Quantam chemistry program exchange, Bloomington, Indiana Program No. 395. In: Molecular Mechanics. Burkert U Allinger NL (eds.), ACS Monograph 177, Washington DC, USA.

24. Talete srl (2007) DRAGON Plus for windos software for molecular descriptor calculations version 5.5, Milano, Italy.

25. Laszlo T (2007) QSAR studies regarding the inhibition of the carbonic anhydrase by the sulfonamides containing a picolinoyl group. Revista De Chimie 58: 191-194.

26. Singh S (2009) Comparative QSAR studies on the novel series of thiazolones and tetrazole derivatives as HCV NS5B polymerase allosteric inhibitors. Lett. Drug Des. Discov 6: 286-297.

27. Singh S, Singh S, Shukla P (2010) Modeling of novel HIV-1 protease inhibitors incorporating $\mathrm{N}$ - Aryl-oxazolidinone-5-carboxamides as P2 ligands using quantum chemical and topological finger print descriptors. Med. Chem. Res 20: $1556-1565$

28. PRECLAV and DESCRIPT programes are available from Center of Organic Chemistry Bucharest-Romanian Academy.

29. Golbraikh A, Tropsha A (2002) Beware of q 2!. Journal of molecular graphics and modelling 20: 269-276.

30. Osten DW (1988) Selection of optimal regression models via cross-validation Journal of Chemometrics 2: 39-48.

31. OECD (2007) Organization for Economic Co-operation and Development Guidance Document on the Validation of (Quantitative) Structure-Activity Relationship QSAR Models. OECD Document ENV/JM/ MONO.

32. Netzeva TI, Worth AP, Aldenberg T, Benigni R, Cronin MT, et al. (2005) Current status of methods for defining the applicability domain of (quantitative) structure-activity relationships. ATLA 33: 155-173.

33. Tropsha A, Gramatica P, Gombar VK (2003) The importance of being earnest: validation is the absolute essential for successful application and interpretation of QSPR models. Molecular Informatics 22: 69-77.

34. Weaver S, Gleeson MP (2008) The importance of the domain of applicability in QSAR modeling. Journal of Molecular Graphics and Modelling 26: 1315-1326.

35. NCSS (2004) Statistical Software Delux package, 329 North 1000 East; Kaysville, UT, USA.

36. Dennis RC (1982) Residuals and Influence in Regression. New York: Chapman, Todeschini HR, Lasagni M, Marengo E, J. Chemom. 8: 263-273.

37. Todeschini R, Gramatica P (1998) 3D QSAR in Drug Design. Kubinyi H, Folkers G, Martin YC, Kluwer/ESCOM, Dordrecht, The Netherlands 355-380. 